\title{
Prevalência dos tipos de fissura em pacientes com fissuras labiopalatinas atendidos em um Hospital Pediátrico do Nordeste brasileiro
}

\section{Prevalence of kinds of cleft lip and palate at a Pediatric Hospital in Northeast of Brazil}

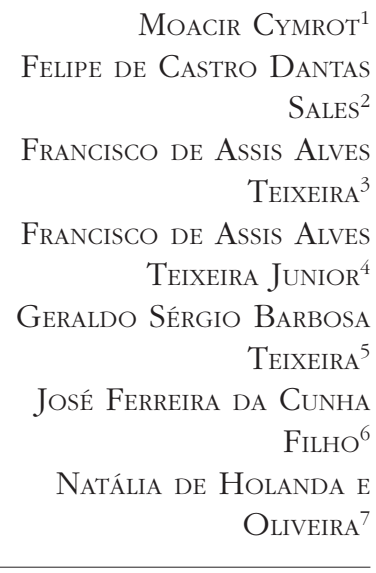

Trabalho realizado no Núcleo de Atendimento Integrado ao Fissurado do Hospital Infantil Albert Sabin (NAIF/HIAS), Fortaleza, CE, Brasil.

Artigo submetido pelo SGP (Sistema de Gestão de Publicações) da RBCP.

Artigo recebido: 6/10/2010 Artigo aceito: 22/11/ 2010

\begin{abstract}
RESUMO
Objetivos: O objetivo deste estudo foi avaliar a prevalência das fissuras labiais e palatais e verificar quais os tipos dessas fissuras que mais acometem as crianças no nosso meio. Método: A amostra é constituída de 551 crianças portadoras de fissura labiopalatinas que procuraram o Núcleo de Atendimento Integrado ao Fissurado do Hospital Infantil Albert Sabin, na cidade de Fortaleza, Ceará, no período de junho de 2008 a fevereiro de 2010. As variáveis estudadas foram sexo, tipo de fissura e lado afetado. Os tipos de fissura foram avaliados segundo a classificação de Spina. As informações foram armazenadas em um banco de dados e submetidas à análise estatística descritiva. Resultados: Cinquenta e três por cento da população amostral era do sexo masculino. Dentre todos os tipos de fissuras labiopalatinas, as fissuras transforame foram as mais frequentes $(72,6 \%)$. Com relação ao lado acometido, as fissuras unilaterais esquerdas foram as mais frequentes $(61,3 \%)$. Observou-se maior prevalência de fissura pós-forame no sexo feminino e de fissura transforame no sexo masculino.
\end{abstract}

Descritores: Fissura palatina. Fissura labial. Incidência.

\begin{abstract}
Objectives: The aim of this study was to assess the prevalence of cleft lip and palate in a Pediatric Center of Northeast of Brazil and to determine which types of these conditions that most affect these children. Methods: From June 2008 to February 2010, 551 children with cleft lip and palate followed by the NAIF (Ceara, Northeast of Brazil) were chosen to participate of the present study. The analyzed variables were sex, type of cleft lip and affected side of the lip. Cleft types were evaluated according to the Spina classification. All information was stored in a database and subjected to descriptive statistical analysis. Results: Fifty-three percent of the assessed population were male. Among all types of cleft lip and palate, the trans-incisive foramen clefts were the most frequent (72.6\%). Regarding the affected side, left clefts were most common (61.3\%). A higher prevalence of trans-incisive foramen cleft in males and post-incisive foramen cleft in girls was observed.
\end{abstract}

Keywords: Cleft Palate. Cleft lip. Incidence.

\footnotetext{
1. Doutor em Cirurgia Plástica; Professor Adjunto da Universidade Estadual do Ceará, Fortaleza, CE, Brasil.

2. Graduando em Medicina; Acadêmico de Medicina da Universidade Estadual do Ceará, Fortaleza, CE, Brasil.

3. Especialista em Cirurgia Plástica e Craniomaxilofacial; Médico assistente do Hospital Albert Sabin, Fortaleza, CE, Brasil.

4. Graduando em Medicina; Acadêmico de Medicina da Universidade Federal do Ceará, Fortaleza, CE, Brasil.

5. Especialista em Cirurgia Plástica; Médico assistente do Hospital Albert Sabin, Fortaleza, CE, Brasil.

6. Especialista em Cirurgia Bucomaxilofacial; Médico assistente do Hospital Albert Sabin, Fortaleza, CE, Brasil.

7. Graduanda em Enfermagem, Fortaleza, CE, Brasil.
} 


\section{INTRODUÇĀO}

As fissuras labiopalatinas são uma das mais frequentes anomalias congênitas orofaciais. Essas malformações acometem o terço médio da face, sendo ocasionadas pela não fusão dos ossos maxilares, durante a sexta e a décima semana de vida intra-uterina ${ }^{1}$. Podem ser um achado isolado ou ocorrer em associação com outros distúrbios, como um componente de uma síndrome ${ }^{1,2}$.

Estudos epidemiológicos têm sido realizados em todo o mundo, e têm mostrado que a prevalência de fissuras labiopalatinas varia muito em relação aos países, sendo de apenas $1,07 \%$, no Japão, e de 4,3\%, em Taiwan ${ }^{3,4}$. No Brasil, a prevalência varia de 0,47 e 1,54 a cada 1.000 nascidos vivos ${ }^{5,6}$.

Várias são as classificações utilizadas para tipificar as fissuras labiopalatinas; entretanto, a mais utilizada pelos profissionais é a classificação de Spina (Quadro 1).

\begin{tabular}{|c|c|c|}
\hline \multicolumn{3}{|c|}{ Quadro 1 - Classificação dos tipos de fissura labiopalatina. } \\
\hline \multirow[t]{3}{*}{$\begin{array}{l}\text { Spina et al. } \\
\text { (1979) }\end{array}$} & $\begin{array}{l}\text { Fissuras pré-forame } \\
\text { incisivo }\end{array}$ & $\begin{array}{l}\text { São as fissuras labiais } \\
\text { unilateral, bilateral e mediana }\end{array}$ \\
\hline & $\begin{array}{l}\text { Fissuras transforame } \\
\text { incisivo }\end{array}$ & $\begin{array}{l}\text { São as de maior gravidade, } \\
\text { unilaterais ou bilaterais, } \\
\text { atingindo lábio, arcada } \\
\text { alveolar e todo palato }\end{array}$ \\
\hline & $\begin{array}{l}\text { Fissuras pós-forame } \\
\text { incisivo }\end{array}$ & $\begin{array}{l}\text { São fissuras palatinas, em } \\
\text { geral medianas, que podem } \\
\text { situar-se apenas na úvula, ou } \\
\text { nas demais partes do palato } \\
\text { duro e mole }\end{array}$ \\
\hline
\end{tabular}

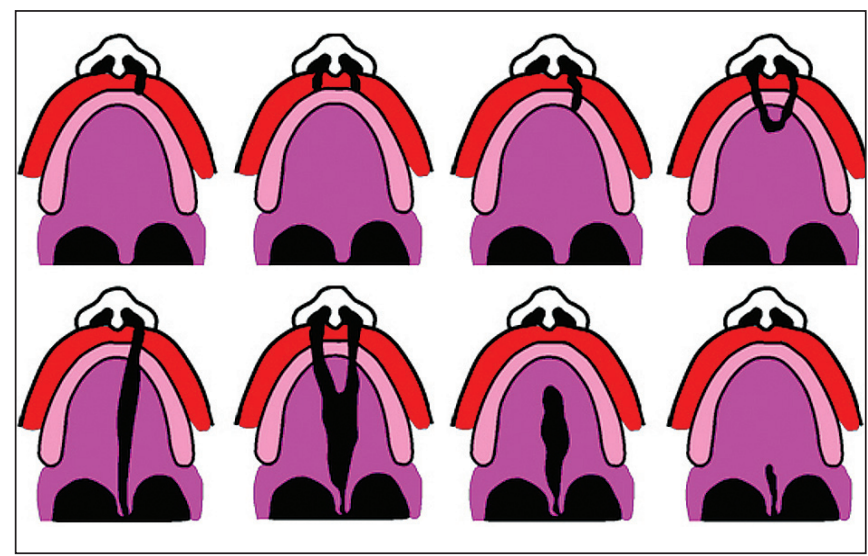

Figura 1 - As malformações labiopalatinas estão representadas da esquerda para direita e de cima para baixo nesta ordem: fissura pré-forame unilateral incompleta, fissura pré-forame bilateral incompleta, fissura pré-forame unilateral completa, fissura préforame completa bilateral, fissura transforame unilateral, fissura transforame bilateral, fissura pós-forame completa e fissura pósforame incompleta.

\section{MÉTODO}

Um estudo retrospectivo foi realizado para identificar todos os pacientes com diagnóstico de fissura labial e/ou palatina e submetidos a tratamento cirúrgico no Hospital Infantil Albert Sabin (HIAS), no período compreendido entre junho de 2008 a fevereiro de 2010.

Uma amostra de 551 crianças foi selecionada, e as variáveis coletadas foram o sexo do paciente e o tipo de anomalia orofacial. As deformidades foram categorizadas baseando-se em uma classificação anatômico-descritiva, tendo como ponto de referência o forame incisivo: fissura pré-forame unilateral incompleta, fissura pré-forame bilateral incompleta, fissura pré-forame completa bilateral, fissura transforame unilateral, fissura transforame bilateral, fissura pós-forame completa, fissura pós-forame incompleta (Figura 1) e fissura palatina submucosa.

\section{RESULTADOS}

Em relação ao sexo dos pacientes com fissura labial e/ ou palatina, verificou-se que $53 \%$ eram do sexo masculino (Tabela 1).

Tabela 1 - Frequência em número e porcentagem de fissura labiopalatina por sexo.

\begin{tabular}{l|c|c} 
Sexo & Frequência & Percentual \\
\hline Masculino & 292 & $53 \%$ \\
Feminino & 259 & $47 \%$ \\
\hline Total & 551 & $100 \%$ \\
\hline
\end{tabular}

Tabela 2 - Frequência em número e porcentagem dos tipos de fissura labiopalatina.

\begin{tabular}{l|c|c}
\hline Tipos de fissura labiopalatina & Frequência & Percentual \\
\hline Fissura pós-forame completa & 128 & $23,2 \%$ \\
Fissura pós-forame incompleta & 2 & $0,4 \%$ \\
Fissura pré-forame bilateral incompleta & 1 & $0,2 \%$ \\
Fissura pré-forame unilateral incompleta & 15 & $2,7 \%$ \\
Fissura submucosa & 5 & $0,9 \%$ \\
Fissura transforame bilateral & 136 & $24,7 \%$ \\
Fissura transforame unilateral & 264 & $47,9 \%$ \\
\hline Total & 551 & $100,0 \%$ \\
\hline
\end{tabular}

Tabela 3 - Frequência do tipo de fissura labiopalatina, de acordo com o sexo.

\begin{tabular}{l|c|c}
\hline Tipos de fissura labiopalatina & \multicolumn{2}{|c}{ Sexo } \\
\hline Fissura pós-forame completa & Feminino & Masculino \\
Fissura transforame bilateral & $79(61,7 \%)$ & $49(38,3 \%)$ \\
Fissura transforame unilateral & $113(42,2 \%)$ & $84(61,8 \%)$ \\
Outros casos & $15(65,2 \%)$ & $151(57,2 \%)$ \\
\hline
\end{tabular}




\begin{tabular}{l|c|c}
\hline \multicolumn{3}{c}{ Tabela 4 - Frequência em número e porcentagem } \\
da uni ou bilateralidade da fissura labial.
\end{tabular}

Tabela 5 - Frequência em número e porcentagem da lateralidade da fissura labial unilateral.

\begin{tabular}{l|c|c}
\hline Localização da deformidade & Frequência & Percentual \\
\hline Unilateral direita & 103 & $36,9 \%$ \\
Unilateral esquerda & 176 & $63,1 \%$ \\
\hline Total & 279 & $100 \%$ \\
\hline
\end{tabular}

Observou-se maior ocorrência da fissura transforame unilateral $264(47,9 \%)$ e, em menor frequência, a fissura transforame bilateral $136(24,7 \%)$, seguida da fissura pósforame completa $128(23,2 \%)$.

Os tipos de fissuras labiopalatinas foram reagrupados em: fissura transforame (fissura transforame bilateral e unilateral), 400 casos $(72,6 \%)$; fissura pós-forame (fissura pós-forame completa e incompleta), 130 casos $(23,6 \%)$; fissura pré-forame (fissura pré-forame bilateral incompleta e unilateral incompleta), 16 casos $(2,9 \%)$ e, finalmente, a fissura submucosa, representando cinco casos e correspondendo a $0,9 \%$ de todos os casos, que também apresentaram fissura de úvula (Tabela 2).

Em relação à fissura transforame bilateral, observouse que a maioria $(84 / 61,8 \%)$ ocorreu em pacientes do sexo masculino. De modo idêntico, pode-se observar em relação à fissura transforame unilateral, correspondendo a $151(57,2 \%)$ casos (Tabela 3$)$.

As fissuras labiais unilaterais e bilaterais correspondem, respectivamente, a $279(67,1 \%)$ e $137(32,9 \%)$ dos casos (Tabela 4).

Dos 279 casos de fissura labial unilateral, independente de serem completas ou incompletas, pré ou transforame, $176(63,1 \%)$ eram do lado esquerdo e $103(36,9 \%)$ do lado direito (Tabela 5).

\section{DISCUSSÃO}

As fissuras palatinas foram mais frequentes no sexo masculino. No presente estudo, não houve diferença estatisticamente significativa na incidência de fissura pré-forame incompleta (fissura labial isolada) entre os sexos, assim como nos estudos de Antoszewski \& Kruk-Jeromin ${ }^{7}$ e Natsume et al. ${ }^{8}$, que observaram que não houve dimorfismo sexual na incidência de fissura labial. No entanto, Fernández et al. ${ }^{9} \mathrm{e}$ Collares et al. ${ }^{10}$, que não observaram dimorfismo sexual na incidência de fissura isolada de lábio. Curiosamente, o estudo realizado por Sullivan ${ }^{11}$ não demonstrou predominância por sexo em nenhum grupo de fissura, enquanto Kumar et al. ${ }^{12}$ encontraram predominância no sexo masculino de todos grupos de fissura.

Em relação ao tipo de fissura, observou-se que a fissura transforame foi a mais comumente encontrada, o que está de acordo com alguns trabalhos encontrados na literatura ${ }^{13-15}$.

A maior prevalência de fissura pós-forame no sexo feminino foi demonstrada, o que é reforçado por outros estudos $^{16,17}$.

Observamos que o lado esquerdo foi o mais acometido pelas fissuras. A literatura consultada é unânime em afirmar que o lado esquerdo é realmente o mais acometido ${ }^{16,18}$.

\section{CONCLUSÃO}

Embora o estudo tenha se baseado em uma população de portadores de fissura labiopalatina submetida a tratamento, os resultados não diferem da maioria dos estudos baseados em nascidos vivos, em que as fissuras transforame são as mais prevalentes dentre todos os tipos. As fissuras esquerdas são mais frequentes que as fissuras direitas; e as fissuras transforame são mais prevalentes no sexo masculino, enquanto as pós-forame prevalecem no sexo feminino.

\section{REFERÊNCIAS}

1. Carreirão S, Lessa S, Zanini AS. Embriologia da face. In: Tratamento das fissuras labiopalatinas. 2a ed. Rio de Janeiro: Revinter;1996. p.1-12.

2. Gorlin R, Cohen M, Hannekam R. Syndromes of the head and neck. $4^{\text {th }}$ ed. New York:Oxford University Press;2001.

3. Imaizumi Y, Yamamura H, Nishikawa M, Matsuoka M, Moriyama I. The prevalence at birth of cogenital malformations at a maternity hospital in Osaka City, 1948-1990. Jinrui Idengaku Zasshi. 1991;36(3):275-87.

4. Shi LM, Chia SE, Chan OY, Chew SK, Foong BH. Prevalence of birth defects and parental work in Singapore live births from 1994 to 1998: a population-based study. Occup Med (Lond). 2002;52(6):325-31.

5. Souza JMP, Buchalla CM, Laurenti R. Estudo da morbidade e da mortalidade perinatal em maternidades. III. Anomalias congênitas em nascidos vivos. Rev Saúde Pública. 1987;21:5-12.

6. Nagem Filho H, Moraes N, Rocha RGF. Contribuição para o estudo da prevalência das más formações congênitas lábio-palatais na população escolar de Bauru. Rev Fac Odontol São Paulo. 1968;6:111-28.

7. Antoszewski B, Kruk-Jeromin J. Epidemiology of cleft lip and palate in Lodz, Poland, in the years 1981-1995. Acta Chir Plast. 1997;39(4):109-12.

8. Natsume N, Kawai T, Ogi N, Yoshida W. Maternal risk factors in cleft lip and palate: case control study. Br J Oral Maxillofac Surg. 2000;38(1):23-5.

9. Fernández JMT, Medlich MAM, Díaz MAS, Estrada NLV. Fisura labiopalatina. Análisis epidemiológico de 121 pacientes. Rev ADM. 1993;50(3):165-7.

10. Collares MVM, Westphalen ACA, Costa TCD, Goldim JR. Fissuras lábio-palatinas: incidência e prevalência da patologiano Hospital de Clínicas de Porto Alegre. Um estudo de 10 anos. Rev AMRIGS. 1995;39(3):183-8.

11. Sullivan WG. Cleft lip with and without cleft palate in blacks: an analysis of 81 patients. Plast Reconstr Surg. 1989;84(3):406-8.

12. Kumar P, Hussain MT, Cardoso E, Hawary MB, Hassanain J. Facial clefts 
in Saudi Arabia: an epidemiologic analysis in 179 patients. Plast Reconstr Surg. 1991;88(6):955-8.

13. Furlaneto EC, Pretto SM. Estudo epidemiológico dos pacientes atendido no Serviço de Defeitos de Face da PUCRS. Rev Odonto Ciência. 2000;15(29):39-56.

14. Graziosi MAOC, Salgado MAC, Castilho JCM. Investigação epidemiológica em indivíduos portadores de fendas labiais e/ou palatinas. PGR: Pós-Grad Rev Fac Odontol São José dos Campos. 2000;3(1):81-7.

15. Baroneza JE, Faria MJSS, Kuasne H, Carneiro JLV, Oliveira JC. Dados epidemiológicos de portadores de fissuras labiopalatinas de uma insti- tuição especializada de Londrina, Estado do Paraná. Acta Sci Health Sci. 2005;27(1):31-5.

16. França CMC, Locks A. Incidência das fissuras lábio-palatinas de crianças nascidas na cidade de Joinville (SC) no período de 1994 a 2000. J Bras Ortodont Ortoped Fac. 2003;8(47):429-36.

17. Al Omari F, Al-Omari IK. Cleft lip and palate in Jordan: birth prevalence rate. Cleft Palate Craniofac J. 2004;41(6):609-12.

18. Bunduki V, Ruano R, Sapienza AD, Hanaoka BY, Zugaib M. Diagnóstico pré-natal de fenda labial e palatina: experiência de 40 casos. Rev Bras Ginecol Obstet. 2001;23(9):561-6.

\section{Correspondência para:}

Moacir Cymrot

Rua Bonfim Sobrinho, 316 apto. 901 - Bairro Fátima - Fortaleza, CE, Brasil - CEP 60040-500

E-mail: moacymrot@uol.com.br 